\title{
Quantitative infrared micro-thermography of light emitting diodes
}

\author{
by K. S. Chang*, J.-Y. Kim*, M. H. Kook*, S. Y. Ryu*, S. C. Yang*, and G. H. Kim*
}

*Division of Instrument Development, Korea Basic Science Institute, 113 Gwahangno, Yusung-gu, Daejeon 305-333, Republic of Korea, ksc@kbsi.re.kr

\begin{abstract}
We reports on the method to measure precise temperature distribution on the GaN-based light-emitting diodes in wafer level by using quantitative infrared micro-thermography. By analyzing, extracting, and correcting the factors, which deteriorate the accuracy of temperature distribution measurement of LED, we were able to determine a precise temperature distribution on the biased LED surface from the measured infrared thermal radiation distribution. From the experimental results, we were able to observe from where the local self-heat generated and how it distributes on the emitting surface of LEDs
\end{abstract}

\section{Introduction}

Nowadays, infrared micro-thermography is used in various applications where spatially highly resolved temperature distribution measurements are required [1-3], because it has the advantage of non-contact, fast measurement speed, and high spatial and thermal resolution. However, infrared micro-thermography has been rarely used in precise temperature mapping of LEDs due to the accuracy limitation. Precise temperature measurement of LEDs using infrared micro-thermography is influenced by many factors, including emissivity and reflectivity uncertainty of various materials on the LED surface, radiation from ambient and measurement system itself, and uncertainty of infrared optical transmission and detector response. These factors discredit the accuracy of temperature distribution measurement of LEDs by using infrared micro-thermography. In this study, we analyze the factors, which deteriorate the accuracy of temperature measurement, and propose the algorithm to compensate the factors to achieve high precision temperature distribution measurement of LEDs using infrared micro-thermography.

\section{Experiment and Results}

Figure 1 shows the schematic diagram and optical microscope image of the InGaN/GaN multiple quantum well (MQW) green LED with lateral electrode. For the temperature distribution measurement by infrared micro-thermography, the LED wafer was epilayer-up bonded to the copper heat sink using silver paste and then they were bonded on the thermoelectric cooler (TEC) mount. A resistance temperature detector (RTD) was placed on the dummy sapphire wafer attached in close proximity to the LED wafer on the same TEC mount side-by-side for the measurement and control of LED wafer temperature. A blackpaint with an emissivity of 0.96 , measured with emissometer, was painted on the empty surface of LED wafer to be the reference point and on the dummy sapphire wafer for the calibration measurement.

To measure the thermal radiation distribution on the LED surface, we used infrared microscope system, which consists of infrared thermal imaging camera (640x512 cooled InSb IRFPA, $15 \mu \mathrm{m}$ pixel pitch) and microscope objective lens manufactured by FLIR Systems Inc. The infrared microscope system has a spectral response in the 3.5-5.1 $\mu \mathrm{m}$ range, a noise equivalent temperature difference (NETD) of $30 \mathrm{mK}, 5 x$ magnification with instantaneous field of view of 3 $\mu \mathrm{m}$, and a working distance of $19 \mathrm{~mm}$.

For the same measuring situation during both calibration and LED imaging, calibration is carried out on the highly emissive black-painted area of dummy sapphire wafer loaded in close proximity to the LED wafer on the TEC mount, instead of calibration using commercial extended area blackbody. equation:

The output signal of an infrared image sensor in micro-thermography system can be described with following

$$
\begin{aligned}
I_{s}[T(x, y)] & =R(x, y)\left\{\varepsilon_{s}(x, y) I_{b b}[T(x, y)]+r_{s}(x, y) I_{a m b}(x, y)+I_{b a c k}(x, y)\right\} \\
& =R(x, y)\left\{\varepsilon_{s}(x, y) I_{b b}[T(x, y)]+I_{r}(x, y)\right\}+I_{o f f s e t}(x, y)
\end{aligned}
$$

where $R(x, y)$ is the spatial response variation of the instrument, including detector response and optical transmission characteristics, $\varepsilon_{S}(x, y)$ is sample emissivity distribution, which combined with Plank's blackbody radiation, $I_{b b}(x, y)$, represents the emission from the sample, $r_{s}(x, y)$ is the sample surface reflectivity, which combined with ambient radiation incident on the sample surface, $I_{a m b}(x, y)$, represents the reflected radiation, $I_{r}(x, y)$, and $I_{b a c k}(x, y)$ includes all background radiation, that reaches the IRFPA, its main component being the stray radiation emitted from surrounding outside the field of view and inside the micro-thermography system itself. Accordingly, to extract a precise temperature distribution from the measured infrared thermal radiation distribution on the LED surface, we have to determine the 
response image $R(x, y)$, superimposed offset signal image $I_{\text {offset }}(x, y)$, emissivity map $\varepsilon_{s}(x, y)$, and reflected radiation signal image $I_{r}(x, y)$. From measured infrared thermal images of black-painted area on dummy sapphire wafer at two different temperatures, we extracted non-uniform response image of instrument and superimposed offset radiation image. To obtain the emissivity map and reflected radiation image on the LED surface, two infrared images at different temperatures of LED wafer were measured. After correcting the response and superimposed offset signal images from the measured infrared thermal images of LED wafer, emissivity map and reflected radiation image were computed. By correcting these factors from the measured infrared thermal radiation image of LED, we were able to extract a precise absolute temperature image of the biased LEDs and hence able to observe from where the local self-heat generated and how it distributes on the emitting area of LEDs, as shown in figure 2. This procedure does not require prior knowledge of the material parameters and also instrument characteristics. By comparing the absolute temperature and electroluminescence (EL) intensity images of LEDs, it is found that strongly localized overheating near the p-contact edge on the LED surface at the high injection current level is due to the local Joule heating and increased nonradiative recombination of carriers near the $\mathrm{p}$-contact edge, which are caused by high current and photon density in the vicinity of p-contact, as a result of current crowding.. This quantitative infrared micro-thermography method can be used in the future to improve the performance of LEDs by optimizing the design of LEDs.

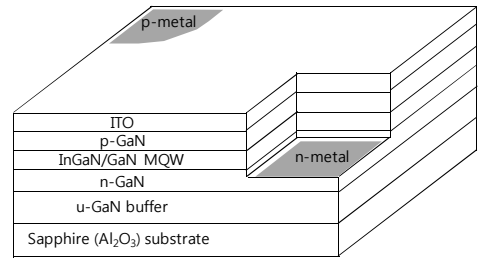

(a)

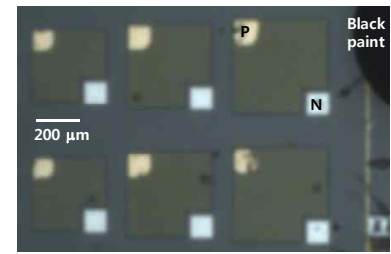

(b)

Fig. 1. (a) Schematic diagram of the InGaN/GaN MQW green LED structure. (b) Top view of optical microscope image of the fabricated LED wafer under test
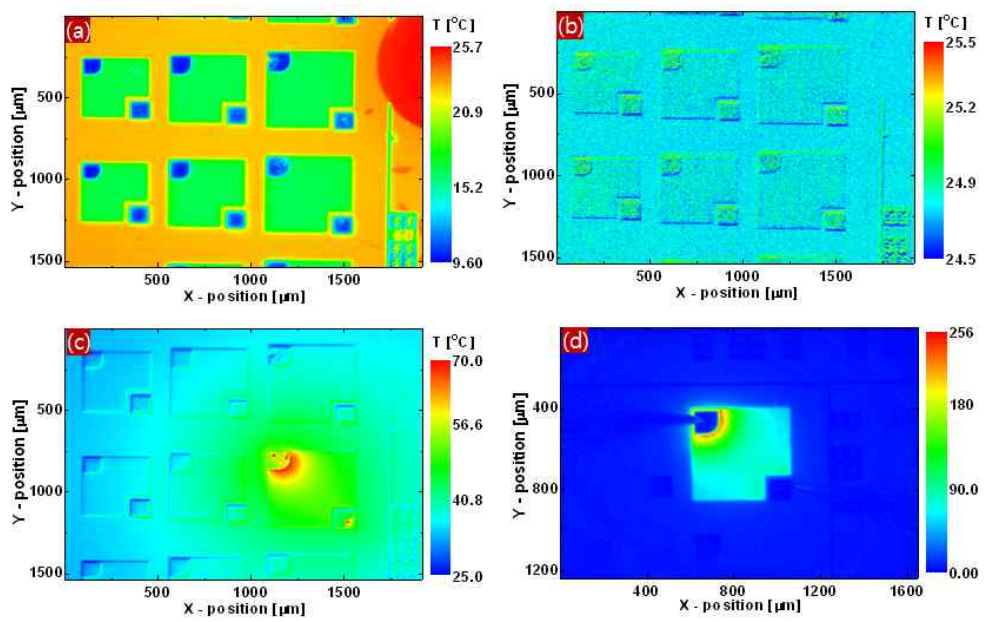

Fig. 2. (a) Non-corrected and (b) corrected temperature image of LED wafer without bias. (c) Precise surface temperature image and (c) EL intensity image of LED with cw bias current of $200 \mathrm{~mA}$. The temperature of heat sink was kept at $25^{\circ} \mathrm{C}$.

\section{REFERENCES}

[1] M. Freitag, H-Y. Chiu, M. Steiner, V. Perebeinos and P. Avouris, "Thermal infrared emission from biased graphene", Nature Nanotech. 5, 497-501, 2010.

[2] R. Furstenberg, C. A. Kendziora, S. V. Stepnowski and R. A. McGill, "Infrared microthermography of microfabricated devices", Rev. Sci. Instrum. 78, 064903, 2007.

[3] J. LeClech, M. Ziegler, J. Mukherjee, J. W. Tomm, T. Elsaesser, J-P. Landesman, B. Corbett, J. G. Mclnerney, J. P. Reithmaier, S. Deubert, A. Forchel, W. Nakwaski, and R. P. Sarzala, "Microthermography of diode lasers: The impact of light propagation on image formation", J. Appl. Phys. 105, 014502, 2009.

[4] A. Kozlowska, M. Latoszek, J. W. Tomm, F. Weik, T. Elsaesser, M. Zbroszczyk, M. Bugajski, B. Spellenberg, and M. Bassler, "Analysis of thermal images from diode lasers: Temperature profiling and reliability screening", Appl. Phys. Lett. 86, 203503, 2005. 